resistenze agli antimicrobici, con lo scopo di ottenere indicazioni utili al clinico per un uso razionale degli antibiotici.

Materiali e Metodi Sono state analizzate le emocolture (3 prelievi) relative al triennio 2002-04. Sono stati inclusi nello studio solo i patogeni isolati da almeno due prelievi diversi e di un sicuro patogeno anche da un solo prelievo nel corso dello stesso episodio infettivo, con esclusione dei duplicati. L'esame dei campioni è stato eseguito col sistema Bactec 9240 (Becton Dickinson), l'identificazione e l'antibiogramma dei batteri con il sistema Microscan Walk Away (DadeBehring). La tipizzazione dei lieviti è stata eseguita con ApiCan (BioMerieux) e l'antimicogramma con Sensititre Yeastone (Trek Diagnostic System). La sensibilità è stata valutata secondo i criteri del NCCLS.

Risultati Delle 1.133 batteriemie, 484 sono state sostenute da gram positivi (42,7\%), 448 da gram negativi (39,5\%), 62 da miceti $(5,5 \%), 35$ da anaerobi $(3,1 \%)$ e 104 erano miste $(9,2 \%)$. I flaconi contaminati sono stati 625 su un totale di $57.910(1,07 \%)$. Tra i gram positivi sono stati isolati 193 S.aureus (39,9\% del totale) 89 S.epidermidis (18,4\%), 51 E.faecalis/faecium (10,5\%), 50 S.pneumoniae (10,3\%), 32 streptococchi viridanti $(6,6 \%)$ e $69(14,3 \%)$ gli altri. Tra i gram negativi 244 erano E.coli $(54,5), 113$ organismi del gruppo KES $(25,2 \%)$ e 91 altri $(20,3 \%)$. La sensibilità degli isolati è risultata del 53,8\% all'Ampicillina e dell' $80,1 \%$ alla Ciprofloxacina di E.coli, del $92,5 \%$ alla Ciprofloxacina di K.pneumoniae, del 71,4\% all'Imipenem di P.aeruginosa per lo più multiresistente, alla Meticillina del $72,1 \%$ di S.aureus e del $25,5 \%$ di S.epidermidis, rispettivamente del $100 \%$ e del $43,1 \%$ all'Ampicillina di E.faecalis e E.faecium, del $100 \%$ ai glicopeptidi degli enterococchi e di S.aureus, $81,8 \%$ alla Penicillina di S.pneumoniae. Tredici ceppi di gram negativi erano produttori di ESBL e 5 ceppi di SCN erano resistenti ai glicopeptidi (3 alla Vancomicina e 2 alla Teicoplanina).

Conclusioni È da segnalare un aumento della resistenza alla Meticillina di S.aureus e alla Penicillina di S.pneumoniae, un'alta incidenza di S.epidermidis meticillino resistenti, l'isolamento di SCN resistenti a glicopeptidi e la bassa frequenza di ceppi multiresistenti di gram negativi, costituiti per lo più da $P$. aeruginosa.

\title{
049
}

\section{EMOCOLTURE NEL TRIENNIO 2002-2004: PREVALENZA E ANTIBIOTICO RESISTENZA DEI MICRORGANISMI ISOLATI}

\author{
Sartori R., Menghini L.G., Ober P., Caola I., Caciagli P. \\ Laboratorio di Microbiologia Ospedale di Trento
}

Scopo Valutare i microrganismi che sono più frequentemente isolati dalle emocolture nel nostro laboratorio e le relative 